\title{
Robust finite-time control of descriptor Markovian jump systems with impulsive
}

Xiaoming $\mathrm{Su}^{1}$ and Xiujie Zhao ${ }^{1 *}$ (D)

"Correspondence:
zxjbzwz@163.com
${ }^{1}$ School of Science, Shenyang
University of Technology, Shenyang,
China

China

\section{照 Springer}

\begin{abstract}
In this paper, we obtain sufficient conditions ensuring stability of the robust finite-time for descriptor Markovian jump systems with impulsive effects and time-varying norm-bounded disturbance, especially, when the system is in actuator saturation. Using the theory of Lyapunov functions and the concept of convex hull-based representation of saturation function. We design the state feedback controller and obtain estimation of domain of attraction, extending the results to convex optimization problems; the solvability condition of the controller can be equivalent to a feasibility problem of coupled linear matrix inequalities (LMIs). Finally, we present some numerical examples showing the effectiveness of the obtained theoretical results.
\end{abstract}

Keywords: Descriptor Markovian jump systems; Actuator saturation; Finite-time control; Impulsive systems

\section{Introduction}

Descriptor systems, also called singular systems, generalized state-space systems, or differential-algebraic systems, have been widely used in many scientific areas because they can better describe the actual system. Descriptor system theory has become an important field in the study of modern control theory. When the structural parameters of a system are randomly mutated, it is naturally modeled as a Markovian jump system or a semi-Markov jump system. Markovian jump systems can be regarded as an extension of single-mode systems to multimodal systems with essentially more complex structure than single-mode systems. During the last decades, Markovian jump systems have attracted great attention in the field of control because they are more suitable for dynamic systems with random changes in the structure of model than single-mode systems. They are widely used in some practical systems, such as manufacturing systems, power systems, economic systems, spare systems, and many other systems [1,2]. Hence a great number of fundamental notions and substantive results are also emerging. The authors in [3] investigated the stochastic admissibility problems for descriptor Markovian jump systems with partially unknown transition rates, descriptor Markovian jump systems with time-varying delay, and nonlinear descriptor Markovian jump systems with time delay. The problems of the robust exponential stability of uncertain singular Markovian jump time-delay systems were studied in [4]. Shen, Su, and Park [5, 6] extended passive and nonfragile fault detection filtering problem, which is investigated for a class of discrete-time singular Markov

(c) The Author(s) 2019. This article is distributed under the terms of the Creative Commons Attribution 4.0 International License (http://creativecommons.org/licenses/by/4.0/), which permits unrestricted use, distribution, and reproduction in any medium, provided you give appropriate credit to the original author(s) and the source, provide a link to the Creative Commons license, and indicate if changes were made. 
jump systems (SMJs) with time-varying delays. Their attention is focused on the design of a general filter that contains the mode-independent and mode-dependent parts to address the filtering issue and on the design of a mode-dependent nonfragile fault detection filter to guarantee the fault detection system to be stochastically admissible with an $H_{\infty}$ performance index for all admissible uncertainties. In [7] a reliable filtering is designed so that the considered filtering error system in the presence of a time-varying delay and sensor failures is mean-square exponentially admissible with a specified decay rate and simultaneously satisfies an $H_{\infty}$ performance.

In addition, finite-time stability means that the system is stable in finite or short time, which is first mentioned in [8]. Based on the Lyapunov theory, the transient performance of the finite-time interval internal system is dealt with. Some researchers [9-12] give a definition of finite-time stabilization and finite-time boundedness. The time-domain stability is a special form of time-domain boundedness, and time-domain boundedness is an extended concept of time-domain stability. They are interrelated and different from each other. During the last decades, people paid more attention to the bounded problem of the system state within a limited time. With the advance of time and linear matrix inequality (LMI) technology, scholars had a deeper understanding of the stability of the time domain of a dynamic system and obtained some meaningful results about the stability of the time domain. In particular, [13] focuses on the problem of robust finite-time stabilization for one family of uncertain singular Markovian jump systems. Sufficient conditions for singular stochastic finite-time boundedness are obtained for a class of singular stochastic systems with parametric uncertainties and time-varying norm-bounded disturbance.

Impulsive systems are a kind of discontinuous systems. The impulsive phenomenon exists in different fields of nature and evolutionary processes, which states sudden changes at some points. It is a transient change of state at a certain time in the actual system. The impulsive effect can better describe the evolution process of the system state. From the control point of view, its influence on the stability of the pulse can be divided into two categories, namely, suppression of the stability of an unstable pulse and improvement of the stability of a stable pulse. It is worth mentioning that there have been some important results in time-domain stability for Markov jump systems with impulses. In [14] a new concept of stochastic finite-time stability for a class of nonlinear Markovian switching systems with impulsive effects is introduced. In [15] a stochastic finite-time stability (SFTS) and control synthesis for a class of nonlinear Markovian jump stochastic systems with impulsive effects is proposed. The impulsive of a system can be better described by introducing a time-varying stochastic Lyapunov function with discontinuities at impulse times.

Actuator saturation [16-20] means that if the input of the system actuator reaches a certain limit, then it enters the saturation state. Because further increasing the input cannot affect the output of the actuator, the saturation of the actuator reduces the dynamic performance of the system and even leads to instability of the closed-loop system. Therefore, it is necessary to study the saturation problem. In [21] the robust stochastic problem for discrete-time uncertain singular Markov jump systems with actuator saturation is considered. In [22] the problem of robust exponential stabilization for uncertain impulsive bilinear time-delay systems with saturating actuators is investigated. In [23] the problems of robust linear feedback stabilization and estimation of domain of attraction for a class of uncertain impulsive systems with saturating actuator are investigated. 
It can be seen from analysis of the previous literature that, in spite of many studies about the finite-time stability of Markovian jump systems, there are no papers on finite-time stability of systems with actuator saturation and impulse and disturbance effects, which is important and significant in engineering applications. Motivated by these, in this paper, we consider the finite-time stability of systems with simultaneous impulse and saturation effects. Sufficient conditions for the time-domain stability of the system are given by Lyapunov function theory, free weight matrix, LMI, and S-procedure. Based on the previous conditions, a state feedback controller is designed so that the resultant closed-loop system is finite-time stable. Finally, an example is given to solve the problem by MATLAB.

\section{Notations}

Throughout the paper, for real symmetric matrices $X$ and $Y$, the notation $X>Y$ means that the matrix $X-Y$ is positive definite; $I$ is the identity matrix of appropriate dimension; the superscript $\mathrm{T}$ represents the transpose; $\operatorname{diag}\{\cdots\}$ denotes a block-diagonal matrix. For a symmetric block matrix, we use $*$ as an ellipsis for the terms that are introduced by symmetry; $\mathrm{E}\{\cdot\}$ denotes the expectation operator with respect to given probability measure $P$.

\section{Modeling}

Given a complete probability space $(\Omega, F, P)$, the continuous-time descriptor Markovian jump impulsive system is described by

$$
\begin{aligned}
& E \dot{x}(t)=(A(r(t))+\Delta A(r(t))) x(t)+(B(r(t))+\Delta B(r(t))), \\
& \operatorname{sat}(u(t))+G(r(t)) \omega(t), \quad t \neq t_{k}, \\
& x_{k}^{+}(t)=A_{d, k} x(t), \quad t=t_{k}, \\
& x\left(t_{0}\right)=x_{0}, \quad r\left(t_{0}\right)=r_{0}, \quad k=1,2, \ldots, \\
& y(t)=C(r(t)) x(t)+D(r(t)) \operatorname{sat}(u(t))+M(r(t)) \omega(t),
\end{aligned}
$$

where $x(t) \in R^{n}$ is the state vector, $y(t)=R^{m}$ is the control output, $u(t) \in R^{m}$ is the control input, $E \in R^{n \times n}$ is a descriptor matrix with $\operatorname{rank}(E)=r \leq n, A(r(t)), B(r(t)), C(r(t))$, $D(r(t)), M(r(t))$, and $G(r(t))$ are known matrices of appropriate dimensions depending on $r(t)$, where $\{r(t), t \geq 0\}$ is a continuous-time Markovian stochastic process defined on a probability space and taking values in a finite space; its transition probabilities from mode $i$ at time $t$ to mode $j$ at time $t+1$ are described as

$$
P(r(t+\delta)=j \mid r(t)=i)= \begin{cases}\gamma_{i j}+o(\delta) & \text { if } j \neq i, \\ 1+\gamma_{i j} \delta+o(\delta) & \text { if } j=i,\end{cases}
$$

where $\delta>0, \lim _{t \rightarrow 0}(o(\delta) / \delta)=0, \gamma_{i j} \geq 0(i, j \in S, j \neq i)$ is the transition rate from $i$ to $j$, and $\gamma_{i i}=-\sum_{j \in S, j \neq i} \gamma_{i j}$. The saturating function sat: $R^{p}-R^{p}$ is defined as

$$
\begin{aligned}
& \operatorname{sat}(u(t))=\left[\operatorname{sat}\left(u_{1}(t)\right) \operatorname{sat}\left(u_{2}(t)\right) \cdots \operatorname{sat}\left(u_{p}(t)\right)\right]^{T}, \\
& \operatorname{sat}\left(u_{i}(t)\right)=\operatorname{sign}\left(u_{i}(t)\right) \min \left\{1,\left|u_{i}(t)\right|\right\} .
\end{aligned}
$$


In the case of unity saturation level, that is, $\operatorname{sat}\left(u_{i}(t)\right) \leq 1, i=1,2, \ldots, p ; \Delta A(r(t))$ and $\Delta B(r(t))$ are matrix functions with time-varying uncertainties. Further,

$$
[\Delta A(r(t)) \quad \Delta B(r(t))]=H_{e}(i) \Delta(t, i)\left[F_{a}(i) \quad F_{b}(i)\right]
$$

where $H_{e}(i), F_{a}(i), F_{b}(i)$ are the known real constant matrices of appropriate dimensions, and $\Delta(t, i)$ is an unknown analytic function matrix with Lebesgue-measurable elements satisfying

$$
\Delta(t, i)^{T} \Delta(t, i) \leq I
$$

If (2) and (3) are established, then $\Delta A(r(t))$ is called the structural robust uncertainty, and $\Delta B(r(t))$ is said to be permissible.

Moreover, the disturbance $\omega(t)$ satisfies

$$
\int_{o}^{\infty} \omega^{T}(t) \omega(t) d t \leq d, \quad d \geq 0
$$

For a matrix, we denote the $j$ th row of $F(i)$ as $f_{i j}$ and define $L(F(i))$ as

$$
L(F(i))=\left\{x(t) \in R^{n}:\left|f_{i j} x(t)\right| \leq 1, j=1,2, \ldots, p\right\} .
$$

Let $P \in R^{n \times n}$ be a symmetric matrix such that $E^{T} P E \geq 0$ and define the set

$$
\Omega\left(E^{T} P E\right)=\left\{x(t) \in R^{n}: x^{T}(t) E^{T} P E x(t) \leq 1\right\} .
$$

Let $D$ be the set of $p \times p$ diagonal matrices whose diagonal elements are either 1 or 0 . Suppose that each element of $D$ is labeled as $D_{l}, l=1,2, \ldots, 2^{p}$ and denote $D_{l}^{-}=I-D_{l}$, Clearly, if $D_{l} \in D$, then $D_{l}^{-} \in D$.

\section{Definition 1}

1. The continuous-time system (1) is said to be uniformly regular if there is a constant $s$ such that the characteristic polynomial $\operatorname{det}(s E-A(r(t)))$ is not identically 0 for any $t \in[0, T]$.

2. The continuous-time system (1) is said to be impulse free in the time interval $[0, T]$ if $\operatorname{deg}(\operatorname{det}(s E-A(r(t))))=\operatorname{rank}(E)$ for all $t \in[0, T]$.

Definition 2 Given three positive scalars $c_{1}, c_{2}$, $T$ with $c_{1}<c_{2}$, positive definite matrices $R_{i}, i \in S$, and positive definite matrix-valued functions $\Gamma_{i}$, a descriptor Markovian jump impulsive system is finite-time stable with respect to $\left(c_{1}, c_{2}, T, R_{i}, \Gamma_{i}\right)$ if

$$
x^{T}(0) E^{T} R_{i} E x(0) \leq c_{1} \quad \Rightarrow \quad x^{T}(t) E^{T} \Gamma_{i} E x(t)<c_{2} \quad \forall t \in[0, T]
$$

for all admissible uncertainties satisfying (2).

Definition 3 ([13]) Let $V(x(t), r(t), t)$ be a stochastic Lyapunov function of a closed-loop SMJS. We define the operator $J$ by

$$
J V(x(t), r(t), t)
$$




$$
\begin{aligned}
= & \lim _{\Delta t \rightarrow 0} \frac{1}{\Delta t}\{\mathrm{E}\{V(x(t+\Delta t)), r(t+\Delta t), \\
& t+\Delta t \mid x(t)=x, r(t)=i\}-V(x(t), r(t), t)\} \\
= & V_{t}(x(t), r(t), t)+V_{x}(x(t), r(t), t) \\
& +\sum_{j=1}^{k} \pi_{i j} V(x(t), r(t), t) .
\end{aligned}
$$

Lemma 1 ([24]) Let $F(i), H(i) \in R^{p \times n}$. Then for any $x(t) \in L(H(i))$,

$$
\operatorname{sat}(F(i) x(t)) \in \operatorname{co}\left\{D_{i} F(i) x(t)+D_{j}^{-} H(i) x(t), j=1,2, \ldots, 2^{p}\right\}
$$

or, equivalently,

$$
\operatorname{sat}(F(i) x(t)) \in \sum_{l=1}^{2^{p}} a_{l}(t)\left(D_{l} F(i)+D_{l}^{-} H(i)\right) x(t)
$$

where co stands for the convex hull, $a_{l}, l=1,2, \ldots, 2^{p}$, are some scalars satisfying $0 \leq a_{l} \leq 1$ and $\sum_{l=1}^{2^{p}} a_{l}=1$.

Lemma 2 ([25]) Given a set of suited dimension real matrices $T_{1}, T_{2}$, and $F(t)$ is a timevarying matrix with $F(t)^{T} F(t) \leq I$, Then, we have the following:

(1) For any scalar $\varepsilon>0$,

$$
T_{1} F(t) T_{2}+T_{2}^{T} F(t)^{T} T_{1}{ }^{T} \leq \varepsilon T_{1} T_{1}{ }^{T}+\varepsilon^{-1} T_{2}{ }^{T} T_{2} .
$$

(2) For any positive definite matrix G,

$$
T_{1} T_{2}+T_{2}^{T} T_{1}^{T} \leq T_{1} G T_{1}{ }^{T}+T_{2}^{T} G^{-1} T_{2}
$$

Lemma 3 ([22]) Let $v(t)$ be a nonnegative function such that

$$
v(t) \leq a+b \int_{0}^{t} v(s) d s, \quad 0 \leq t \leq T
$$

for some constants $a, b \geq 0$. Then we have the following inequality:

$$
v(t) \leq a e^{b t}, \quad 0 \leq t \leq T .
$$

In this paper, we consider the state feedback controller

$$
u(t)=K(r(t)) x(t)
$$


such that the closed-loop system is defined by

$$
\begin{aligned}
& E \dot{x}(t)=\sum_{l=1}^{2^{p}} a_{l}(t)\left(\bar{A}_{i} x(t)+\bar{B}_{i}\left(D_{l} K_{i}+D_{l}^{-} H_{i}\right) x(t)+G_{i} \omega(t)\right), \quad t \neq t_{k}, \\
& x_{k}^{+}(t)=A_{d, k} x(t), \quad t=t_{k}, \\
& x\left(t_{0}\right)=x_{0}, \quad r\left(t_{0}\right)=r_{0}, \quad k=1,2, \ldots, \\
& y(t)=\sum_{l=1}^{2^{p}} a_{l}(t)\left(C_{i} x(t)+D_{i}\left(D_{l} K_{i}+D_{l}^{-} H_{i}\right) x(t)+M_{i} \omega(t)\right) .
\end{aligned}
$$

For convenience, denote the matrix $A(r(t))$ as $A_{i}$ and

$$
\bar{A}_{i}=\bar{A}(r(t))=A(r(t))+\Delta A(r(t)), \quad \bar{B}_{i}=\bar{B}(r(t))=B(r(t))+\Delta B(r(t)) .
$$

\section{Main results}

\subsection{Robust finite-time stabilization}

Theorem 1 Consider the closed-loop system (5) for $t \in[0, T]$. Let $c_{1}, c_{2}, T$ be three positive scalars with $c_{1}<c_{2}$, let $R_{i}, i \in S$ be positive definite matrices, and let $\Gamma_{i}$ be positive definite matrix-valued functions, Suppose that there exist a scalar $\alpha \geq 0$, a set of nonsingular matrices $P_{i} \in R^{n \times n}$, two sets of symmetric positive definite matrices $Q_{2}(i) \in R^{d \times d}, i \in S$, and $Q_{1}(i) \in R^{n \times n}, i \in S$, such that the following hold:

$$
\begin{aligned}
& E^{T} P_{i}=P_{i}^{T} E \geq 0, \\
& {\left[\begin{array}{cc}
A_{l}^{T}(i) P_{i}+P_{i}^{T} A_{l}(i)+\varepsilon P_{i}^{T} H_{e}(i) H_{e}^{T}(i) P_{i}+ & \\
\varepsilon^{-1} F_{l}^{T}(i) F_{l}(i)+\sum_{j=1}^{2^{p}} \pi_{i j} E^{T} P_{j}-\alpha E^{T} P_{i} & P_{i}^{T} G_{i} \\
* & -Q_{2}(i)
\end{array}\right]<0,} \\
& A_{d, k}^{T} E^{T} P_{i} A_{d, k}-E^{T} P_{i}<0, \quad t=t_{k}, \\
& E^{T} \Gamma_{i} \leq E^{T} P_{i} \leq E^{T} R_{i}, \\
& \lambda_{\max }\left(Q_{1}(i)\right) c_{1} e^{\alpha t}+d \lambda_{\max }\left(Q_{2}(i)\right) e^{\alpha t}<c_{2} \lambda_{\min }\left(Q_{1}(i)\right),
\end{aligned}
$$

where

$$
\begin{gathered}
\bar{A}_{i}+\bar{B}_{i} \operatorname{sat}\left(u_{i}\right)=A_{i}+\Delta A_{i}+\left(B_{i}+\Delta B_{i}\right)\left(D_{l} K_{i}+D_{l}{ }^{-} H(i)\right) \\
\quad=A_{l}(i)+H_{e}(i) \Delta(t, i) F_{l}(i), \\
A_{l}(i)=A_{i}+B_{i}\left(D_{l} K_{i}+D_{l}^{-} H(i)\right), \\
F_{l}(i)=F_{a}(i)+F_{b}(i)\left(D_{l} K_{i}+D_{l}^{-} H(i)\right)
\end{gathered}
$$

for all $i, j=1,2, \ldots, s, l=1,2, \ldots, 2^{n}$, and $\Omega\left(E^{T} X_{i} E\right) \subset L\left(H_{i}\right)$. Then the closed-loop system (5) with respect $\left(c_{1}, c_{2}, T, R, d, \Gamma_{i}\right)$ is robust finite-time stable within $\bigcap_{i=1}^{N} \Omega\left(E^{T} X_{i} E\right)$.

Proof Firstly, we prove that the closed-loop systems (5) is regular and impulse-free in the time interval $[0, T]$. By the Schur complement and condition (7) we obtain

$$
A_{l}^{T}(i) P_{i}+P_{i}^{T} A_{l}(i)+\left(\pi_{i j}-\alpha\right) E^{T} P(i)<-\sum_{j=1, j \neq i}^{2^{p}} \pi_{i j} E^{T} P_{j} \leq 0 .
$$


Choose nonsingular matrices $M$ and $N$ such that

$$
\begin{aligned}
& M E N=\left[\begin{array}{ll}
I_{r} & 0 \\
0 & 0
\end{array}\right], \quad M A_{l}(i) N=\left[\begin{array}{ll}
A_{11}(i) & A_{12}(i) \\
A_{21}(i) & A_{22}(i)
\end{array}\right], \\
& M P(i) N=\left[\begin{array}{ll}
P_{11}(i) & P_{12}(i) \\
P_{21}(i) & P_{22}(i)
\end{array}\right] .
\end{aligned}
$$

Then, according to (6) and (12), it is not difficult to prove that $P_{12}(i)=0$ and $\operatorname{det}\left(P_{22}(i)\right) \neq 0$. Pre- and post-multiplying (11) by $N^{T}$ and $N$, we get

$$
\begin{aligned}
& N^{T} A_{l}^{T}(i) P_{i} N+N^{T} P_{i}^{T} A_{l}(i) N+\left(\pi_{i i}-\alpha\right) N^{T} E^{T} P_{i} N<0 \\
& \left(M A_{l}(i) N\right)^{T}\left(M P_{i} N\right)+\left(M P_{i} N\right)^{T}\left(M A_{l}(i) N\right)+\left(\pi_{i i}-\alpha\right)(M E N)^{T}\left(M P_{i} N\right)<0 \\
& {\left[\begin{array}{ll}
A_{11}^{T}(i) P_{11}(i) & A_{12}^{T}(i) P_{22}(i) \\
A_{21}^{T}(i) P_{11}(i) & A_{22}^{T}(i) P_{22}(i)
\end{array}\right]+\left[\begin{array}{ll}
P_{11}^{T}(i) A_{11}(i) & P_{11}^{T}(i) A_{12}(i) \\
P_{22}^{T}(i) A_{21}(i) & P_{22}^{T}(i) A_{22}(i)
\end{array}\right]} \\
& \quad+\left(\pi_{i i}-\alpha\right)\left[\begin{array}{cc}
P_{11}(i) & 0 \\
0 & 0
\end{array}\right]<0 .
\end{aligned}
$$

We can easily obtain that $A_{22}^{T} P_{22}(i)+P_{22}^{T} A_{22}(i)<0$ and $A_{22}(i)$ is nonsingular, which implies that the closed-loop SMJS is regular and impulse-free in the time interval $[0, T]$.

Construct the following Lyapunov function: $V(x(t), i)=x^{T}(t) E^{T} P_{i} x(t)$.

When $t \neq t_{k}$, using Definition 3, we obtain that

$$
\begin{aligned}
\ell V(x(t), i)= & \dot{x}^{T}(t) E^{T} P_{i} x(t)+x^{T}(t) E^{T} P_{i} \dot{x}(t)+x^{T}(t)\left(\sum_{j=1}^{N} E^{T} P_{j}\right) x(t) \\
= & (E \dot{x}(t))^{T} P_{i} x(t)+x^{T}(t) P_{i}{ }^{T} E \dot{x}(t)+x^{T}(t)\left(\sum_{j=1}^{N} E^{T} P_{j}\right) x(t) \\
= & x^{T}(t)\left[\bar{A}_{i}^{T} P_{i}+P_{i}{ }^{T} \bar{A}+\left(D_{l} K_{i}+D_{l}^{-} H_{i}\right)^{T} \bar{B}_{i}^{T} P_{i}\right. \\
& \left.+P_{i}{ }^{T} \bar{B}_{i}\left(D_{l} K_{i}+D_{l}^{-} H_{i}\right)+\sum_{j=1}^{N}\left(E^{T} P_{j}\right)\right] x(t) \\
& +\omega^{T}(t) G^{T}(i) P_{i} x(t)+x^{T}(t) P^{T}{ }_{i} G(i) \omega(t)<0,
\end{aligned}
$$

where $z(t)=\left[\begin{array}{c}x(t) \\ \omega(t)\end{array}\right]$, so that

$$
\ell V(x(t), i)=z^{T}(t)\left[\begin{array}{cc}
\left(\bar{A}_{i}^{T}+D_{l} K_{i}+D_{l}^{-} H_{i}^{T} \bar{B}_{i}^{T}\right) P_{i+} & P_{i}^{T} G_{i} \\
P_{i}^{T}\left(\bar{A}_{i}+\bar{B}_{i} D_{l} K_{i}+D_{l}^{-} H_{i}\right)+\sum_{j=1}^{2^{p}} \pi_{i j} E^{T} P_{j} & 0 \\
* & 0
\end{array}\right] z(t)
$$


By Lemma 1, Lemma 2, and (2), this formula is equivalent to

$$
\ell V(x(t), i)=z^{T}(t)\left[\begin{array}{cc}
A_{l}^{T}(i) P_{i}+P_{i}^{T} A_{l}(i)+\varepsilon P_{i}^{T} H_{e}(i) H_{e}^{T}(i) P_{i}+ & P_{i}^{T} G_{i} \\
\varepsilon^{-1} F_{l}^{T}(i) F_{l}(i)+\sum_{j=1}^{2^{p}} \pi_{i j} E^{T} P_{j} & 0 \\
* & 0
\end{array}\right] z(t) .
$$

According the last formula and (7), we can obtain that $\ell V(x(t), r(t)=i, t)<0$ and

$$
\ell V(x(t), i)<\alpha V(x(t), i)+\omega^{T}(t) Q_{2}(i) \omega(t)
$$

When the system depends on the state to jump, applying (8), we have

$$
V\left(t, x_{k}^{+}\right)-V(t, x)=x^{T}(t)\left[A_{d, k}^{T} E^{T} P(t) A_{d, k}-E^{T} P(t)\right] x(t)<0 .
$$

So we derive that $V(t, x)$ is strictly decreasing on $T$.

Integrating (13) from 0 to $t$ and using Lemma 3, we have

$$
\begin{aligned}
\mathrm{E}\{V(x(t), i)\} & <V(x(0), i)+\alpha \int_{0}^{t} V(x(s), i) d s+\int_{0}^{t} \omega^{T}(t) Q_{2}(i) \omega(t) d \tau \\
& <V(x(0), i)+\alpha \int_{0}^{t} V(x(s), i) d s+d \lambda_{\max }\left(Q_{2}(i)\right) \\
& <\left[V(x(0), i)+d \lambda_{\max }\left(Q_{2}(i)\right)\right] e^{\alpha t} \\
& <V(x(0), i) e^{\alpha t}+d \lambda_{\max }\left(Q_{2}(i)\right) e^{\alpha t},
\end{aligned}
$$

where $Q_{1}(i)=E^{-T} R_{i}^{-\frac{1}{2}} E^{T} P_{2} R_{i}^{\frac{1}{2}} E^{-1}$, and $\lambda_{\max }\left(Q_{1}(i)\right)$ and $\lambda_{\min }\left(Q_{1}(i)\right)$ are the maximum and minimum eigenvalues of $Q_{1}(i)$. Thus

$$
\begin{aligned}
E\{V(x(t), i)\} & =E\left\{x^{T}(t) E^{T} R_{i}^{\frac{1}{2}} Q_{1}(i) R_{i}^{\frac{1}{2}} E x(t)\right\} \\
& \geq \lambda_{\min } Q_{1}(i) E\left\{x^{T}(t) E^{T} \Gamma_{i} E x(t)\right\} .
\end{aligned}
$$

On the other hand,

$$
\begin{aligned}
V(x(0)) e^{\alpha t} & =x^{T}(0) E^{T} R_{i}^{\frac{1}{2}} Q_{1}(i) R_{i}^{\frac{1}{2}} E x(0) e^{\alpha t} \\
& \leq \lambda_{\max } Q_{1}(i) E^{T} x^{T}(0) R_{i} E x(0) e^{\alpha t} \leq \lambda_{\max } Q_{1}(i) c_{1} e^{\alpha t} .
\end{aligned}
$$

Therefore

$$
E\left\{x^{T}(t) E^{T} \Gamma_{i} E x(t)\right\} \leq \frac{\lambda_{\max }\left(Q_{1}(i)\right) c_{1} e^{\alpha t}+d \lambda_{\max }\left(Q_{2}(i)\right) e^{\alpha t}}{\lambda_{\min }\left(Q_{1}(i)\right)}<c_{2} .
$$

This proves that system (5) is robust finite-time stable.

Theorem 2 Let (5) be a closed-loop system with $\omega(t)=0$ for $t \in[0, T]$, let $c_{1}, c_{2}, T$ be three positive scalars with $c_{1}<c_{2}$, let $R_{i}, i \in S$, be positive definite matrices, and let $\Gamma_{i}$ be positive definite matrix-valued functions. Suppose that there exist a scalar $\alpha \geq 0$, a set of 
nonsingular matrices $P_{i} \in R^{n \times n}$, and two sets of symmetric positive definite matrices $Q_{2}(i) \in$ $R^{d \times d}, i \in S$, and $Q_{1}(i) \in R^{n \times n}, i \in S$, such that the following hold:

$$
\begin{aligned}
& E^{T} P_{i}=P_{i}^{T} E \geq 0, \\
& A_{d, k}^{T} E^{T} P_{i} A_{d, k}-E^{T} P_{i}<0, \quad t=t_{k}, \\
& E^{T} \Gamma_{i} \leq E^{T} P_{i} \leq E^{T} R_{i}, \\
& A_{l}^{T}(i) P_{i}+P_{i}^{T} A_{l}(i)+\varepsilon P_{i}^{T} H_{e}(i) H_{e}^{T}(i) P_{i}+\varepsilon^{-1} F_{l}^{T}(i) F_{l}(i)+\sum_{j=1}^{2^{p}} \pi_{i j} E^{T} P_{j}-\alpha E^{T} P_{i}<0, \\
& \lambda_{\max }\left(Q_{1}(i)\right) c_{1} e^{\alpha t}<c_{2} \lambda_{\min }\left(Q_{1}(i)\right),
\end{aligned}
$$

for all $i, j=1,2, \ldots, s, l=1,2, \ldots, 2^{n}$, and $\Omega\left(E^{T} X_{i} E\right) \subset L\left(H_{i}\right)$. Then the closed-loop system (5) with respect $\left(c_{1}, c_{2}, T, R, d, \Gamma_{i}\right)$ is robust finite-time stable within $\bigcap_{i=1}^{N} \Omega\left(E^{T} X_{i} E\right)$.

This result can be proved in much the same way as Theorem 1 .

\subsection{Robust state feedback controller}

Theorem 1 gives a set of conditions to judge if some initial state is in the domain of attraction in mean square sense. To further facilitate the synthesis procedure, we will state these conditions in terms of LMIs.

Theorem 3 Suppose that, for each mode $i \in S$ and given scalars $\varepsilon_{i}>0$, there exist a positive definite symmetric matrix $X_{i}>0$, matrices $Y_{i}$ and $H_{i}$ for $i=1,2, \ldots, 2^{n}$, and $\Omega\left(E^{T} P_{i} E\right) \subset$ $L\left(H_{i}\right)$. Then for all uncertainties satisfying (2) and (3), the closed-loop system is robust finitetime stable within $\bigcap_{i=1}^{N} \Omega\left(E^{T} X_{i} E\right)$, and the state feedback controller gain matrix is given by $K_{i}=L_{i} X_{i}^{-1}, i=1,2, \ldots, N$.

Let $X_{R} \subset R^{n}$ be a prescribed bounded convex set containing origin that can be represented as the polyhedron $X_{R}=\operatorname{co}\left\{x_{0}^{1}, x_{0}^{2}, \ldots, x_{0}^{q}\right\}$, where $x_{0}^{1}, x_{0}^{2}, \ldots, x_{0}^{q}$ are a priori given initial states in $R^{n}$. To see if the initial states $x_{0} \subset R^{n}$ are in the domain of attraction in the mean square sense, we can formulate the following optimization problem:

$$
\begin{aligned}
& \max _{P_{i}>0, F_{i}, H_{i}, Q_{i}, \varepsilon_{i}} \alpha \\
& \text { s.t. } \quad \text { (i) } \alpha x_{0}^{j} \in \Omega\left(E^{T} P_{i} E\right), \quad j=1,2, \ldots, s, \\
& \text { (ii) Inequalities (7), (10), } \\
& \text { (iii) } \Omega\left(E^{T} P_{i} E\right) \subset L(H(i)),
\end{aligned}
$$

where $h_{i q}$ denotes the $q$ th row of $H_{i}$. If $\max |\alpha|>1$, then $x_{0} \in \Omega\left(E^{T} P_{i} E\right)$. Noticing that the optimization problem is nonconvex, we need to formulate this problem into a convex optimization problem.

Let $P_{i}=X_{i}^{-1}, L_{i}=K_{i} X_{i}, E^{T} P_{i}=P_{i}^{T} E=P_{i}^{-1} H(i) P_{i}^{-T}$. Condition (i) is equivalent to

$$
\alpha^{2}\left(x_{0}^{j}\right)^{T} E^{T} P_{i} E x_{0}^{j} \leq 1, \quad j=1,2, \ldots, s .
$$


By the Schur complement it can be converted to

$$
\left[\begin{array}{cc}
\alpha^{-2} & \left(x_{0}^{j}\right)^{T} E^{T} X_{i} \\
X_{i} E x_{0}^{j} & X_{i}
\end{array}\right] \geq 0, \quad j=1,2, \ldots
$$

Letting $\beta=\alpha^{-2}$, (15) can be rewritten as

$$
\left[\begin{array}{cc}
\beta & \left(x_{0}^{j}\right)^{T} E^{T} X_{i} \\
X_{i} E x_{0}^{j} & X_{i}
\end{array}\right] \geq 0, \quad j=1,2, \ldots
$$

Inequality (7) can be transformed into

$$
\left[\begin{array}{ccc}
\sum & P_{i}^{T} H e(i) & F(i)^{T} \\
* & -\varepsilon_{i}^{-1} I & 0 \\
* & * & \varepsilon_{i} I
\end{array}\right]<0
$$

where

$$
\sum=A_{l}^{T}(i) P_{i}+P_{i}^{T} A_{l}(i)+\sum_{j=1}^{N} \pi_{i j} E^{T} P_{j}-P_{i}^{T} G(t) Q_{2} G(t)^{T} P_{i}-\alpha E^{T} P_{i}
$$

Pre- and postmultiplying (17) by te diagonal matrix $\operatorname{diag}\left\{X_{i}, I, I\right\}$, we obtain

$$
\left[\begin{array}{ccc}
X_{i} \sum X_{i} & X_{i} P_{i}^{T} H e(i) & X_{i} F(i)^{T} \\
* & -\varepsilon_{i}^{-1} I & 0 \\
* & * & \varepsilon_{i} I
\end{array}\right]<0 .
$$

This matrix can be transformed into

$$
\left[\begin{array}{cccc}
\Pi & X_{i} P_{i}^{T} H e(i) & X_{i} F_{i}^{T} & \Pi_{1} \\
* & -\varepsilon_{i}^{-1} I & 0 & 0 \\
* & * & \varepsilon_{i} I & 0 \\
* & * & * & -\Pi_{2}
\end{array}\right]<0,
$$

where

$$
\begin{aligned}
& \Pi=X_{i} A_{m}^{T}(i) P_{i} X_{i}+X_{i} P_{i}^{T} A_{m}(i) X_{i}-X_{i} P_{i}^{T} G(t) Q_{2} G^{T}(t) P_{i}, \\
& \bar{A}_{i}+\bar{B}_{i} \operatorname{sat}\left(u_{i}\right)=A_{i}+\Delta A_{i}+\left(B_{i}+\Delta B_{i}\right)\left(D_{l} L_{i} X_{i}^{-1}+D_{l}^{-} H(i)\right) \\
& =A_{m}(i)+H_{e}(i) \Delta(t, i) F_{m}(i), \\
& A_{m}(i)=A_{i}+B_{i}\left(D_{l} L_{i} X_{i}^{-1}+D_{l}^{-} H(i)\right), \\
& F_{m}(i)=F_{a}(i)+F_{b}(i)\left(D_{l} L_{i} X_{i}^{-1}+D_{l}^{-} H(i)\right), \\
& \Pi_{1}=\left[\sqrt{\pi_{i, 1}} X_{i}, \ldots, \sqrt{\pi_{i, i-1}} X_{i}, \sqrt{\pi_{i, i+1}} X_{i}, \ldots, \sqrt{\pi_{i, k}} X_{i}\right] \\
& \Pi_{2}=\operatorname{diag}\left\{\begin{array}{c}
P^{T}(1) H^{-1}(1) P(1), \ldots, P^{T}(i-1) \\
H^{-1}(i-1) P(i-1) P^{T}(i+1) H^{-1}(i+1) \\
P(i+1), \ldots, P^{T}(k) H^{-1}(k) P(k)
\end{array}\right\}
\end{aligned}
$$


Let $Q_{i}=Q_{1}^{-1}(i)=R_{i}^{-1 / 2} X_{i} R_{i}^{-1 / 2}$ and

$$
\begin{aligned}
& \lambda_{\max }(Q)=\frac{1}{\lambda_{\min }\left(Q_{1}(i)\right)}, \\
& \lambda<\lambda_{\min }\left(Q_{1}(i)\right), \quad \lambda_{\max }\left(Q_{1}(i)\right)>1, \quad \lambda_{\max }\left(Q_{2}(i)\right)>1 .
\end{aligned}
$$

Thus (10) can be rewritten as

$$
\frac{c_{1}+d}{\lambda_{1}}-c_{2} e^{-\partial t}<0
$$

or, equivalently,

$$
\left[\begin{array}{cc}
-c_{2} e^{-\partial t} & \sqrt{c_{1}+d} \\
\sqrt{c_{1}+d} & -\lambda
\end{array}\right]<0 .
$$

Since $\Omega\left(E^{T} P_{i} E\right) \subset L(H(i))$, we have

$$
x^{T}(t) h_{i q}^{T} h_{i q} x(t) \leq x^{T}(t) E^{T} P_{i} E x(t),
$$

which is equivalent to

$$
h_{i q}^{T} h_{i q}-P_{i} \leq 0, \quad i=1,2, \ldots, s, q=1,2, \ldots, m
$$

Using the Schur complements, we have

$$
\left[\begin{array}{cc}
-P_{i} & h_{i q}^{T} \\
* & -I
\end{array}\right] \leq 0, \quad q=1, \ldots, m,
$$

and the optimization problem can be transformed into the following linear matrix inequality problem:

$$
\left\{\begin{array}{l}
\min _{X_{i}>0, Y_{i}, H_{i}} \beta \\
\text { s.t. LMIs (16), (18), (19), and (20), }
\end{array}\right.
$$

where $\varepsilon_{i}>0$ is a given scalar. If $\min \beta<1$, then $x_{0} \in \Omega\left(E^{T} P_{i} E\right)$. The state feedback controller gain $K_{i}=L_{i} X_{i}^{-1}$ can be obtained by solving the linear matrix inequality problem directly.

\section{Simulation example}

Let us consider the robust finite-time stability for system (1) with the following coefficient matrices:

$$
E=\left[\begin{array}{ccc}
2.5 & 5 & 2.5 \\
0 & 1.25 & 1.25 \\
0 & 0 & 0
\end{array}\right]
$$


mode 1:

$$
\begin{aligned}
& A(1)=\left[\begin{array}{ccc}
2 & 2.4 & 1.2 \\
1.8 & 1.1 & 1.1 \\
0.5 & 0.2 & 0.5
\end{array}\right], \quad B(1)=\left[\begin{array}{l}
0.6 \\
0.4 \\
0.1
\end{array}\right], \\
& F_{a}(1)=\left[\begin{array}{lll}
0.05 & 0.06 & 0.02
\end{array}\right], \quad F_{b}(1)=0.03, \\
& H e(1)=\left[\begin{array}{l}
0.01 \\
0.02 \\
0.03
\end{array}\right], \quad W_{1}=\left[\begin{array}{ccc}
0.6 & 0.3 & 1.3 \\
0.5 & -0.2 & 0.25 \\
1 & 1.5 & 0.5
\end{array}\right], \\
& {[\Delta A(1) \quad \Delta B(1)]=\left[\begin{array}{llll}
0.0005 & 0.0006 & 0.0002 \\
0.0010 & 0.0012 & 0.0004 & 0.0003 \\
0.0015 & 0.0018 & 0.0006 & 0.0006
\end{array}\right] ;}
\end{aligned}
$$

mode 2:

$$
\begin{aligned}
& A(2)=\left[\begin{array}{ccc}
1 & -1 & 0 \\
2.25 & 0.75 & 2 \\
1.25 & 0.5 & 0.75
\end{array}\right], \quad B(2)=\left[\begin{array}{c}
1.5 \\
-1 \\
-0.75
\end{array}\right], \\
& F_{a}(2)=\left[\begin{array}{lll}
0.02 & 0.04 & 0.05
\end{array}\right], \quad F_{b}(1)=0.04, \\
& H e(2)=\left[\begin{array}{l}
0.03 \\
0.05 \\
0.06
\end{array}\right], \quad W_{2}=\left[\begin{array}{ccc}
0.2 & 0.3 & 1.3 \\
0.5 & -0.2 & 0.25 \\
1 & 1.5 & 0.5
\end{array}\right], \\
& {[\Delta A(2) \quad \Delta B(2)]=\left[\begin{array}{llll}
0.0006 & 0.0012 & 0.0015 \\
0.0010 & 0.0020 & 0.0025 & 0.0012 \\
0.0012 & 0.0024 & 0.0030 & 0.0024
\end{array}\right] .}
\end{aligned}
$$

Let $c_{1}=1, c_{2}=11, T=3, \alpha=0.1$,

$$
\begin{aligned}
x_{0} & =\left[\begin{array}{c}
-0.56 \\
0.36 \\
0
\end{array}\right], \quad R=\left[\begin{array}{lll}
0 & 0 & 1 \\
0 & 0 & 1 \\
0 & 0 & 1
\end{array}\right], \\
\Gamma_{i} & =\left[\begin{array}{ccc}
1 & 0 & 0 \\
0 & 0.2 & 0 \\
0 & 0 & 0.5
\end{array}\right] .
\end{aligned}
$$

We use the LMI toolbox of MATLAB software to solve the optimization problem $\beta=$ $0.218<1$. The gain of the state feedback controller is:

$$
\begin{aligned}
& K_{1}=\left[\begin{array}{lll}
-1.5983 & -1.5495 & 0.0503
\end{array}\right], \\
& K_{2}=\left[\begin{array}{lll}
-0.4159 & -0.0910 & -0.0306
\end{array}\right] .
\end{aligned}
$$

Then the MATLAB simulation is shown in the following image. 


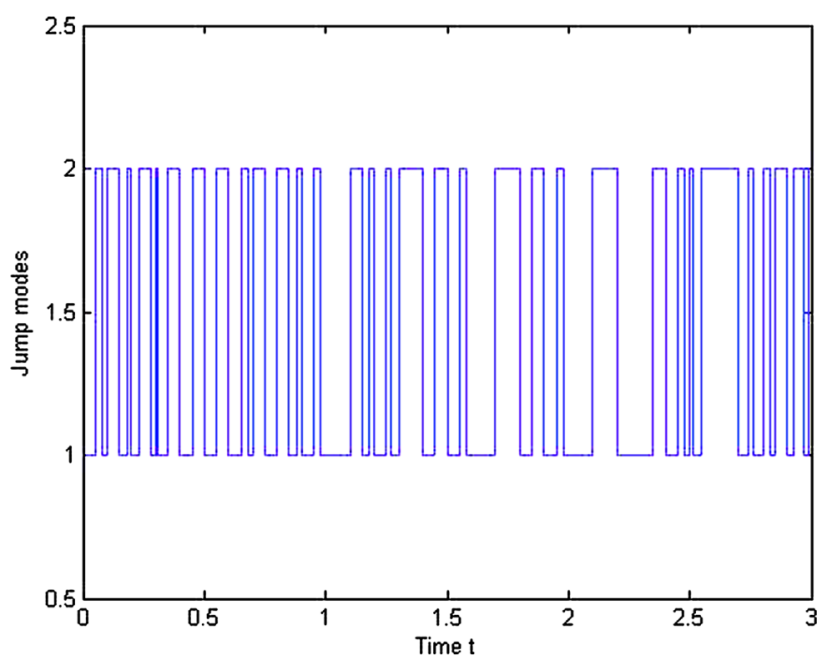

Figure 1 The jumping modes

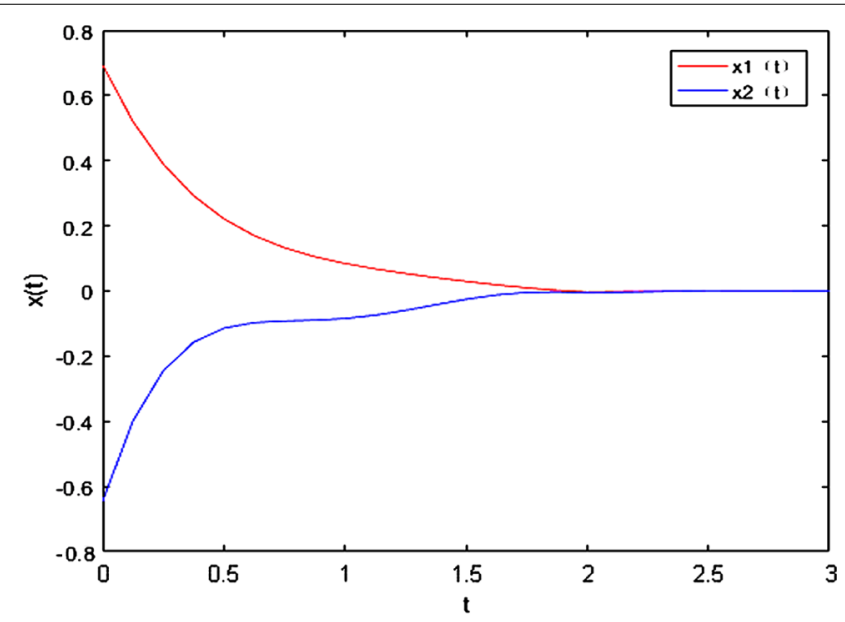

Figure 2 The trajectory of states

One possible realization of Markovian jumping mode is given in Fig. 1. The simulation results under this mode-dependent controller are shown in Fig. 2. We see that the state responses are satisfactory when the saturation appears. The corresponding state trajectory is shown in Fig. 3.

\section{Conclusion}

The robust finite-time control for descriptor Markov jump systems with impulsive effects, actuator saturation, and time-varying norm-bounded disturbance have been investigated. A sufficient condition for the finite-time stability of systems is given according to Lyapunov function theory and LIMs. Based on the conditions above, a state feedback controller is designed such that the resultant closed-loop system is finite-time stable. The simulation results are given by MATLAB. The results in this paper can be applied in communication engineering and other fields. It has important theoretical significance for further study of some problems of descriptor Markovian jump systems (also, see [1-26]). 


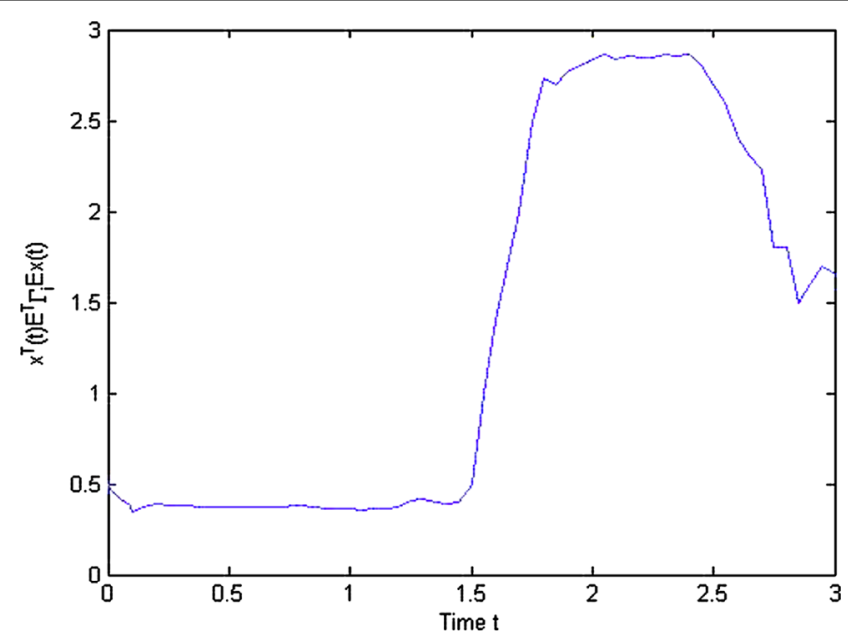

Figure 3 The trajectory of $x^{T}(t) E^{T} \Gamma_{i} E x(t)$

\section{Acknowledgements}

We would like to thank the anonymous referees very much for their valuable comments and suggestions

\section{Funding}

This work was supported by National Natural Science Foundation of China under Grant No. 61074005 and the Talent Project of the High Education of Liaoning province (LR2012005).

\section{Competing interests}

The authors declare that they have no competing interests.

\section{Authors' contributions}

The authors have achieved equal contributions. Both authors read and approved the manuscript.

\section{Publisher's Note}

Springer Nature remains neutral with regard to jurisdictional claims in published maps and institutional affiliations.

Received: 6 November 2018 Accepted: 4 March 2019 Published online: 22 May 2019

\section{References}

1. Feng, J.E., Lam, J., Shu, Z.: Stabilization of Markovian systems via probability rate synthesis and output feedback. IEEE Trans. Autom. Control 55, 773-777 (2010)

2. Wang, Z., Liu, Y., Liu, X.: Exponential stabilization of a class of stochastic system with Markovian jump parameters and mode-dependent mixed time-delays. IEEE Trans. Autom. Control 55, 1656-1662 (2010)

3. Zhang, Q., Liu, C., Zhang, X.: Bifurcations and control in singular biological economic model with stage structure. In: Complexity, Analysis and Control of Singular Biological Systems. Lecture Notes in Control and Information Sciences, vol. 421, pp. 43-66. Springer, London (2012)

4. Zheng-Guang, W.U., Hong-Ye, S.U., Jian, C.H.U.: Robust exponential stability of uncertain singular Markovian jump time-delay systems. Acta Autom. Sin. 36, 558-563 (2010)

5. Shen, H., Su, L., Park, J.H.: Extended passive filtering for discrete-time singular Markov jump systems with time-varying delays. Signal Process. 128, 68-77 (2016)

6. Shen, H., Su, L., Park, J.H.: Robust non-fragile $H_{\infty}$ fault detection filter design for delayed singular Markovian jump systems with linear fractional parametric uncertainties. Hybrid Syst. 32, 65-78 (2019)

7. Liu, G.B., Xu, S.Y., Park, J.H., Zhang, G.M.: Reliable exponential filtering for singular Markovian jump systems with time varying delays and sensor failures. Int. J. Robust Nonlinear Control 28, 4230-4245 (2018)

8. Kamenkov, G.V.: On stability of motion over a finite interval of time. Akad. Nauk SSSR Prikl. Mat. Meh. 17, 529-540 (1953)

9. Dorato, P.: Short time stability in linear time-varying systems. In: Proceedings of the IRE International Convention Record, pp. 83-87 (1961)

10. Kablar, N.A., Debelikovic, D.L.: Finite-time stability of time-varying linear singular systems. Am. Control Conf. IEEE 4 3831-3836 (1994)

11. Garcia, G., Tarbouriech, S., Bernussou, J.: Finite-time stabilization of linear time-varying continuous systems. IEEE Trans. Autom. Control 54, 364-369 (2009)

12. Amato, F., Ariola, M., Cosentino, C.: Finite-time stability of linear time-varying systems: analysis and controller design IEEE Trans. Autom. Control 55, 1003-1008 (2010)

13. Zhang, Y., Liu, C., Mu, X.: Robust finite-time stabilization of uncertain singular Markovian jump systems. Appl. Math. Model. 36, 5019-5121 (2012) 
14. Jia, X., Sun, J., Dong, Y.: Stochastic finite-time stability of nonlinear Markovian switching systems with impulsive effects. J. Dyn. Syst. Meas. Control 134, 011 (2012)

15. Chen, W.H., Wei, C., Lu, X.: Stochastic finite-time stabilization for a class of nonlinear Markovian jump stochastic systems with impulsive effects. J. Dyn. Syst. Meas. Control 137, 044 (2015)

16. Fridman, E., Pila, A., Shaked, U.: Regional stabilization and $\mathrm{H} 1$ control of time-delay systems with saturating actuators. Int. J. Robust Nonlinear Control 13, 885-907 (2003)

17. Zhang, L.X., Boukas, E.-K., Haidar, A.: Delay-range-dependent control synthesis for time-delay systems with actuator saturation. Automatica 44, 2691-2695 (2008)

18. Gomes Da Silva, J.M., Tarbouriech, S.: Local stabilization of discrete-time linear systems with saturating controls: an LMI-based approach. IEEE Trans. Autom. Control 46, 119-125 (2001)

19. Cao, Y.Y., Lin, Z.: Stability analysis of discrete-time systems with actuator saturation by a saturation-dependent Lyapunov function. Automatica 39, 1235-1241 (2003)

20. Alamo, T., Cepeda, A., Limon, D.: Estimation of the domain of attraction for saturated discrete-time systems. J. Astronaut. 69, 274-280 (1998)

21. Ma, S., Zhang, C., Zhu, S.: Robust stability for discrete-time uncertain singular Markov jump systems with actuator saturation. IET Control Theory Appl. 5, 255-262 (2011)

22. Yang, S.J., Shi, B., Zhang, Q., et al.: Robust exponential stabilization of uncertain impulsive bilinear time-delay systems with saturating actuators, 12, 261-265 (2010)

23. Li, Y., Chen, W.H.: Robust stabilization of uncertain impulsive systems with saturating actuator. J. Northwest Normal Univ. Nat. Sci. 6, 10-15 (2013)

24. Hu, T., Lin, Z., Chen, B.M.: Analysis and design for discrete-time linear systems subject to actuator saturation. In: Decision and Control, 2001. Proceedings of the, IEEE Conference on, pp. 97-112. IEEE (2002)

25. Last, E.: Linear matrix inequalities in system and control theory, SAM. In: Proceedings of the IEEE, vol. 86, pp. 2473-2474 (1994)

26. Yan, Z., Zhang, G., Wang, J.: Finite-time stability and stabilization of linear stochastic systems. In: China Control Conference, pp. 1115-1120 (2010)

\section{Submit your manuscript to a SpringerOpen ${ }^{\circ}$ journal and benefit from:}

- Convenient online submission

- Rigorous peer review

- Open access: articles freely available online

- High visibility within the field

Retaining the copyright to your article

Submit your next manuscript at $\boldsymbol{\nabla}$ springeropen.com 